\title{
La medicina genómica en las políticas de salud pública: una perspectiva de investigadores mexicanos del área biomédica
}

\author{
Pablo Francisco Oliva-Sánchez, M en CS, ${ }^{(1,2)}$ Jesús Mario Siqueiros-García, D en F, , $^{(1)}$ \\ José Rogelio Vázquez-González, Lic en Med,(2) Garbiñe Saruwatari-Zavala, M en Bioética, ${ }^{(1)}$ \\ Alessandra Carnevale, Med Esp en Gen. ${ }^{(3)}$
}

Oliva-Sánchez PF, Siqueiros-García JM, Vázquez-González JR, Saruwatari-Zavala G, Carnevale A. La medicina genómica en las políticas de salud pública: una perspectiva de investigadores mexicanos del área biomédica. Salud Publica Mex 2013;55:16-25.

\section{Resumen}

Objetivo. Explorar las perspectivas de los investigadores en medicina genómica y cómo incide esta última en las políticas de salud en México. Material y métodos. Se realizó una investigación cualitativa a partir de entrevistas semiestructuradas, durante el II Congreso Nacional de de Medicina Genómica celebrado en México del 25 al 27 de octubre de 2006. Resultados. Se identificaron cuatro categorías con respecto a la aplicabilidad de la medicina genómica y la viabilidad política de la investigación genómica. Algunos investigadores consideran que las tres "P" de la medicina genómica, predictiva, personalizada y preventiva, generaron "expectativas exageradas" sobre su impacto real en salud pública. Las opiniones se dividieron entre aquéllos que consideran la medicina genómica como el nuevo "paradigma" de salud pública y quienen piensan que se trata más bien de una herramienta de la salud pública. Conclusión. Si bien no cabe duda que la medicina genómica constituye uno de los campos de la salud pública orientados a mejorar las condiones de salud de los mexicanos, no deben perderse de vista sus alcances reales.

Palabras clave: genómica; salud pública; políticas públicas de salud
Oliva-Sánchez PF, Siqueiros-García JM, Vázquez-González JR, Saruwatari-Zavala G, Carnevale A.

Genomic medicine in public health policies:

a perspective of Mexican biomedical researchers.

Salud Publica Mex 2013;55:16-25.

\section{Abstract}

Objective. The purpose of this study was to explore the researcher's perspectives on genomic medicine (GM) and its consequences in the decision-making process regarding the health policy in Mexico. Materials and methods. An exploratory qualitative research was conducted (semistructured questionnaire). The study was conducted during the II National Congress of GM (25 - 27th October 2006). Results. We identified four categories amongst the researcher's perspectives regarding the applicability of GM and the political feasibility of the genomics research. Some researchers believe that the 3P's of GM (Predictive, Personalized, Preventive) have generated "inflated expectations" on the real impact on public health $(\mathrm{PH})$. Opinions were divided among those who believed that GM is the new 'paradigm' of $\mathrm{PH}$ and those who thought that GM is part of a set of tools for $\mathrm{PH}$. Conclusion. To improve the health conditions of the Mexican population, GM must be part in the solution of the $\mathrm{PH}$ problems, but we need a realistic view.

Keywords: genomics; public health; health public policy

(I) Departamento de Estudios Éticos, Jurídicos y Sociales, Instituto Nacional de Medicina Genómica. México, DF, México.

(2) Facultad de Ciencias de la Salud, Universidad Anáhuac México Norte. México, DF, México

(3) Dirección de Investigación, Instituto Nacional de Medicina Genómica. México, DF, México.

Fecha de recibido: II de enero de 20I2 - Fecha de aceptado: 2 I de septiembre de 2012 Autor de correspondencia: Dra. Alessandra Carnevale. Instituto Nacional de Medicina Genómica. Periférico Sur 4809, col. Arenal Tepepan. I46I0 Delegación Tlalpan México, DF, México correo electrónico: acarnevale@inmegen.gob.mx 
$\mathrm{A}$ partir de la publicación en 2001 de los resultados del Proyecto Genoma Humano (PGH), un consorcio de instituciones conformó en México un proyecto para impulsar la medicina genómica $(\mathrm{MG})$, que culminó con la creación del Instituto Nacional de Medicina Genómica (Inmegen) en 2004. ${ }^{1,2}$ El objetivo primordial al introducir la MG en México era contar con aplicaciones predictivas y con tratamientos más efectivos, a la vez que reducir el gasto destinado a los problemas de salud pública (SP). 3,4 De manera paralela al consorcio institucional, diversos proyectos científicos en genética humana en el país introdujeron el concepto de la $\mathrm{MG}^{*}$ en la medicina clínica. ${ }^{5-10}$ En ese contexto, el argumento de que la población mexicana posee una estructura genética particular, producto del mestizaje entre población indígena, española y, en menor proporción, africana, justificó el desarrollo del "primer mapa de variabilidad genómica de la población de México", que se llevó a cabo en el INMEGEN. ${ }^{4,11-13}$ A través de este nuevo enfoque de genómica poblacional se pretende predecir la susceptibilidad de los individuos a desarrollar alguna enfermedad, implementar estrategias preventivas, encontrar blancos terapéuticos específicos e individualizar los tratamientos según las características genéticas de los mexicanos, ya no sólo por sus características clínicas, sino también por su perfil molecular (esto es, se busca llevar a cabo una medicina de mexicanos para mexicanos). 2,4,14 El presente artículo es producto del interés por conocer de una manera más amplia la visión de los investigadores biomédicos mexicanos con respecto al desarrollo de la $\mathrm{MG}$, al igual que vislumbrar el posible impacto de la $\mathrm{MG}$ en las políticas de SP. Se consideró importante entrevistarlos puesto que son ellos quienes saben del estado de los avances científicos y tecnológicos en esta materia, además de que probablemente tenían conocimiento de su aplicabilidad y utilización en el área de la salud . ${ }^{15,16}$ El estudio se llevó a cabo durante el II Congreso Nacional de Medicina Genómica, celebrado en la Ciudad de México del 25 al 27 de octubre de 2006.

\section{Material y métodos}

Se diseñó un estudio cualitativo, descriptivo y exploratorio. De manera contextual, se analizó el punto de

\footnotetext{
* La MG se define como el uso rutinario de análisis genotípicos para mejorar el cuidado de la salud; se basa en la capacidad para conocer la variabilidad genética de cada individuo y modificar el medio donde éste se desarrolla, con el fin de prevenir y tratar enfermedades. La identificación de las variantes genómicas presentes en cada individuo permitirá elaborar perfiles genómicos (pruebas genéticas) y predecir la respuesta de cada paciente a los medicamentos, su predisposición a distintas enfermedades y establecer su pronóstico ante la aparición de complicaciones crónicas.
}

vista de los investigadores biomédicos acerca de la situación actual de la $\mathrm{MG}$, su perspectiva a futuro y su impacto en la política de salud en México. El punto de partida fue la idea de que, al conocer los beneficios que genera la MG, los investigadores desempeñan un papel importante para la inserción de este conocimiento aplicado en el proceso de toma de decisiones en materia de salud. Se realizaron entrevistas individuales, semiestructuradas, basadas en un cuestionario conformado por cuatro preguntas abiertas (cuadro I). El muestreo fue intencional dado que sólo se entrevistó a investigadores participantes en el congreso antes citado. Como criterio de selección se estableció que los entrevistados fuesen investigadores activos en las ciencias genómicas, en genética humana o en genética clínica, y se confirmó su participación en un proyecto de investigación relacionado con la MG. No se entrevistó a investigadores de las áreas éticas, jurídicas y sociales relacionadas con la MG. Las entrevistas tuvieron una duración promedio de 30 minutos; fueron grabadas en cinta de audio casete y posteriormente transcritas. Antes de realizar las entrevistas, se obtuvo el consentimiento informado verbal de cada uno de los entrevistados y se garantizó la confidencialidad de la información.

\section{Análisis de datos}

A partir del discurso vertido en las entrevistas, en la primera etapa de análisis se encontraron tres conceptos que se convirtieron en rectores para la siguiente etapa (cuadro II) donde, gracias a una comparación de posición discursiva llevada a cabo mediante análisis contextual de opiniones, ${ }^{17}$ se identificaron cuatro categorías con una codificación abierta. Se comparó la opinión de los

\section{Cuadro I \\ Cuestionario aplicado a los entrevistados (GuíA DE ENTREVISTA)*}

Pregunta I Desde su opinión jen dónde radica el impacto de la medicina genómica en la salud pública en relación con el control de las enfermedades crónicas?

Pregunta 2 ¿Piensa usted que los organismos públicos y privados que otorgan financiamientos para la investigación en salud en México consideran que la investigación en medicina genómica es una área prioritaria para financiamiento? Sí. No. ¿Por qué?

Pregunta 3 En el futuro, ¿cuál considera usted que será el impacto de la medicina genómica en la salud de los mexicanos?

Pregunta 4 ¿Podría usted dar un ejemplo de una aplicación específica en la práctica médica de las ciencias genómicas, con evidencia comprobada? 
Cuadro II

DEFINICIONES EMERGENTES IDENTIFICADAS EN EL DISCURSO DE LOS INVESTIGADORES MEXICANOS ENTREVISTADOS SOBRE

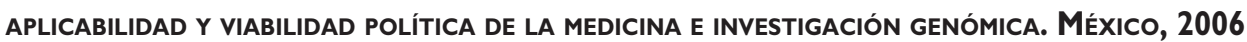

Definición de las 3"p" (discurso de las tres "p": predictiva, personalizada y preventiva)
Nos referiremos al discurso de las 3 "p" de la medicina genómica como el discurso político oficial con el cual se introdujo la medicina genómica en México. Esta última, definida como el uso rutinario de análisis genotípicos para mejorar el cuidado de la salud, se basa en la capacidad de conocer la variabilidad genómica de cada individuo y modificar el medio ambiente en que éste se desarrolla, con el fin de prevenir y tratar enfermedades. La medicina genómica dará como resultado una práctica médica más individualizada (personalizada), predictiva y preventiva. ${ }^{1,2}$

Temporalidad de la aplicación de la medicina genómica

Para hacer el análisis profundo de los discursos se aborda la temporalidad de la aplicabilidad de la MG en la salud. En el corto plazo: cuando se hable de la pronta (5 - 10 años) utilización de los resultados de la medicina genómica en el cuidado de la salud. De largo plazo: se definirá cuando no se prevea de manera inmediata (a más de 10 años) la aplicabilidad de la medicina genómica. ${ }^{2}$

Modernidad

Se considera como la biomolecularización de las actividades preventivas, diagnósticas y terapeúticas relacionadas con la práctica clínica y la salud pública. ${ }^{2,4}$

Fuente: referencias I, 2, 3 y 4

investigadores con respecto a la posición del discurso oficial con el cual se introdujo la MG en México -discurso de las 3 " $p$ "- y se comparó su posición en términos del conocimiento de aplicaciones -médicas y en SP-y la temporalidad en la aparición de las aplicaciones, como resultado del desarrollo científico. Después de realizar la codificación abierta, se identificaron algunas relaciones entre las categorías y se obtuvo por medio de análsis de relaciones, dos temáticas centrales: a) aplicaciones y áreas emergentes de aplicación, y b) factibilidad política: la identificación de instituciones importantes (actores políticos) para el desarrollo de la MG y la inversión.

\section{Resultados}

\section{Categorías de posición discursiva}

En el cuadro III se describen las características de los participantes del estudio. Por otra parte, el resultado de la fase de codificación abierta fue la identificación de las siguientes cuatro categorías:

1. Modernidad inmediata. La categoría hace referencia a la confianza de los investigadores respecto a la temporalidad y presencia de los impactos de la aplicación de la MG, en el corto plazo, en México.

2. Modernidad diferida. Esta categoría está relacionada con la creencia de los investigadores en los objetivos y promesas de la MG, con objetivos de aplicaciones a mediano y largo plazo en términos de temporalidad. Se considera que la MG es una herramienta complementaria en la investigación de los fenómenos de la salud, y su importancia se sustenta en la interacción con otros conocimientos en el campo de la salud.

3. Promesas infladas. Esta categoría hace referencia a que los investigadores consideran que la propuesta de la MG tiene restricciones o limitantes y que sus promesas son exageradas.

4. Escepticismo. La categoría alude a la poca confianza por parte de los entrevistados hacia la aplicación de la MG para resolver problemas de SP.

Cuadro III

Características de los investigadores mexicanos ENTREVISTADOS SOBRE APLICABILIDAD Y VIABILIDAD POLÍTICA DE LA MEDICINA E INVESTIGACIÓN GENÓMICA. MÉxıco, 2006

\begin{tabular}{lc} 
Sexo & $\mathrm{n}(\%)$ \\
Masculino & $\mathrm{II}(68.75)$ \\
\hline Femenino & $5(31.25)$ \\
\hline
\end{tabular}

Profesión

\begin{tabular}{ll} 
Médico clínico & $2(12.50)$ \\
\hline Médico investigador & $9(56.00)$ \\
\hline Investigador no médico & $3(18.75)$ \\
\hline Médico investigador y clínico & $2(12.50)$ \\
\hline
\end{tabular}

S.N.I

\begin{tabular}{ll} 
No & $9(56.00)$ \\
\hline Sí & $7(44.00)$ \\
\hline$n=16$ &
\end{tabular}

SNI: Sistema Nacional de Investigadores 


\section{Análisis de relaciones}

Con el objetivo de tener una dimensión de la interrelación de los ejes temáticos con respecto a las cuatro categorías que representan las posiciones con respecto al discurso de la 3" $p$ " de la MG, se llevó a cabo un análisis de relaciones (cuadro IV).

\section{Modernidad inmediata}

\section{Aplicabilidad y aplicaciones de la $M G$}

En esta categoría los entrevistados manifestaron tener una visión positiva acerca de las promesas de la MG y sus aplicaciones; aceptaron que, bajo el supuesto de una medicina más preventiva, predictiva y personalizada, generará la modernización de la práctica clínica (discurso de las 3 " $p$ " totalmente asumido). Se plantéo que el diagnóstico oportuno, en etapas presintomáticas, por medio de la identificación de biomarcadores genéticos que se asocien con el riesgo de desarrollar enfermedades crónicas, constituirá una aplicación directa de la MG en SP y en las estrategias de la medicina preventiva.

En términos de la temporalidad, los entrevistados consideraron que las aplicaciones se podrán ver en el corto plazo o, incluso, inmediatamente:

.... La MG es, desde mi punto de vista, la herramienta más importante para hacer medicina preventiva y como siempre se ha sabido dentro del ambiente médico, y en la ciencias de la salud, la medicina preventiva es la herramienta real que va a lograr minimizar la prevalencia de las enfermedades crónicas y, por lo tanto, la MG, hoy por hoy, es la herramienta más importante para poder hacer medicina preventiva... el impacto va a ser que va a incidir en el sueño de todos los que estamos en ciencias de la salud, que es que sea una realidad la medicina personalizada (entrevistada E7M).

Se mencionó que el impacto será visible en los programas de tamizaje prenatal -o preconcepcional- de padecimientos crónicos, así como de enfermedades monogénicas. Las áreas donde se promete una aplicación directa y en el corto plazo son la farmacogenómica y la nutrigenómica. Los entrevistados opinaron que la farmacogenómica es el primer paso hacia una medicina personalizada exitosa; uno de ellos se refirió a una prueba farmacogenómica que hoy en día ya se emplea en terapia de anticoagulación en pacientes con cardiopatías o post operados de valvulopatías. Esta prueba se basa en los polimorfismos genéticos de los genes VKORC1, CYP2C9 y CYP4F2.
.... La farmacogenómica es un área muy atractiva si consideramos que puede aumentar la tasa de éxito una vez conocida .... la información genómica del individuo que va a recibir el fármaco... Tenemos recientemente la descripción y la aplicación de información molecular acerca de genes que regulan el metabolismo y la acción de la droga acenocumarina; en este tipo de casos, tenemos variantes en el genoma que determinan el metabolismo de la droga. Con esta información, los médicos podemos prevenir aquellos casos de sangrado importante que se presentan en estos individuos que toman este tipo de droga. Podemos identificar a los individuos y ajustar su dosis del fármaco a la dosis que les corresponde, dependiendo de cómo metabolizan el fármaco (entrevistado E6H).

La nutrigenómica es unárea entendida como "la interrelación estrecha entre alimentación, variantes genómicas y salud." Se considera como una herramienta informativa que puede promover la modificación en el estilo de vida hacia uno más saludable, con el propósito de prevenir, sobre todo, enfermedades cardiovasculares.

\section{Factibilidad política: financiamiento y actores institucionales}

Los entrevistados opinaron que el financiamiento de la MG es prioritario. Subrayaron que es necesario abrir este campo de investigación y que las agencias financiadoras, tanto públicas como privadas, deben invertir en su desarrollo. Empero, no señalaron la manera como tendrían que obtenerse los apoyos económicos y los financiamientos, aunque a lo largo de las entrevistas no dejaron de insistir en la importancia que implica el desarrollo de nuevo conocimiento.

Yo creo que es una de las áreas que debe de estar considerada en los financiamientos de quien dependa, como dice la pregunta, tanto públicos como privados... (entrevistado E6H).

Una de las investigadoras -que en 2006 acababa de llegar a México y se incorporaba a la comunidad científica- opinó acerca de un proceso de monopolización de los financiamientos, tanto públicos como privados, en el área de la genómica por parte de algunos grupos de investigación:

Sí, aunque el problema de los organismos públicos y privados que otorgan financiamiento es que realmente son un monopolio y no se abren a las necesidades prioritarias en el campo de la salud ni a los nuevos grupos de trabajo (entrevistada E7M). 


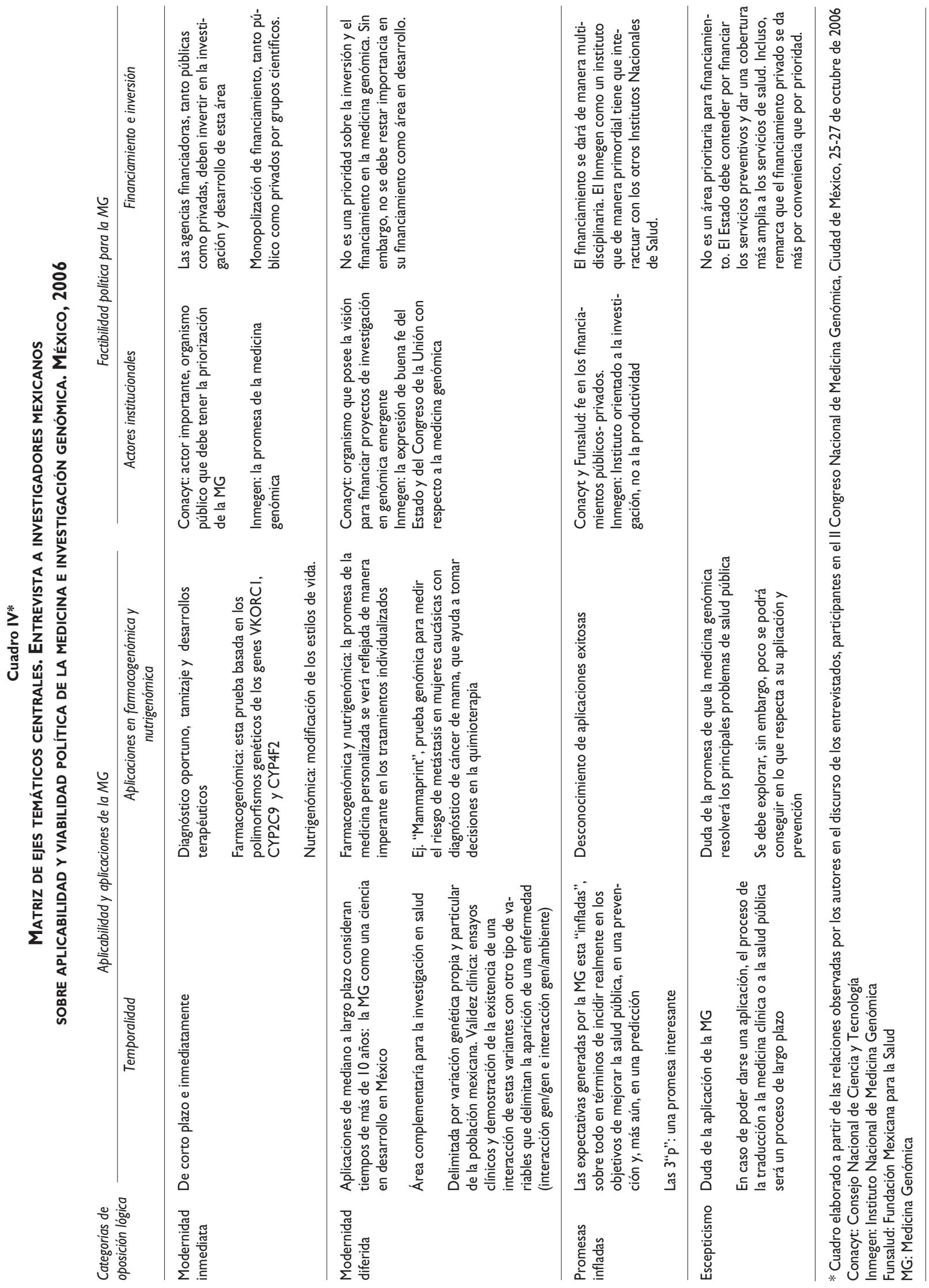


En el discurso de los investigadores se detectaron algunos actores importantes para el desarrollo y consolidación de la MG en México. Se trata de instituciones públicas y / o privadas que desempeñan un papel importante en el financiamiento y la definición de las prioridades en materia de investigación y desarrollo tecnológico, como el Consejo Nacional de Ciencia y Tecnología (Conacyt), institución decisiva en el financiamiento de la investigación. Los investigadores que fueron agrupados en esta categoría en 2006 situaban al Inmegen como un actor nuevo e importante, sobre todo en la consolidación de la genómica aplicada a la salud en México.

...No tengo ahorita en la cabeza cuáles son las áreas prioritarias fundamentalmente de Conacyt, pero debería ser una de las áreas prioritarias (en investigación y desarro1lo)... En un país como el nuestro con pocos recursos para la investigación sobran áreas prioritarias y falta dinero; ése es un problema general (entrevistado E16H).

\section{Modernidad diferida}

\section{Aplicabilidad y aplicaciones de la medicina genómica}

Los entrevistados de esta categoría aceptan el discurso de las 3" $\mathrm{p}^{\prime \prime}$ de la MG y la modernidad que la pone a disposición de la medicina clínica y de la SP. Sin embargo, esta promesa se ve matizada o diferida en dos aspectos: la temporalidad en la aparición de las aplicaciones prácticas, por un lado, y la centralidad de la MG como paradigma médico y en el cuidado de la salud, por el otro. Los investigadores consideraron que la MG tendrá un impacto importante en todas las áreas de la salud, aunque esto será visible sólo en los plazos mediano y largo. La aparición de aplicaciones basadas en conocimiento genómico está definida por diferentes aspectos:

1. Se considera la MG como un área en desarrollo en México, y se definirá al conocer las variantes genéticas propias y particulares de la población nacional, sobre todo aquéllas que se asocien con enfermedades prevalentes.

2. Aunque se asocie una variante genética con una enfermedad, es necesario validar analítica y clínicamente la asociación. Asimismo, es imprescindible demostrar que existe una interacción entre las variantes y otro tipo de variables que contribuyen a la aparición de una enfermedad (interacción gen-gen, interacción gen-ambiente y redes de interacción biológica entre diferentes sistemas). Lo anterior es importante dada la naturaleza compleja de las enfermedades que son objeto de estudio.
3. Para los investigadores, el término "mediano a largo plazo" significa más de 10 años para aplicar la MG a la clínica y a la SP. Una de las investigadoras incluso alargó este plazo: "Yo creo que, desafortunadamente, nos falta ver una visión en unos $30 \mathrm{o}$ 40 años...." (entrevistada E11M).

Por otra parte, los entrevistados opinaron que se necesita una visión multidisciplinaria en las ciencias genómicas en salud y que los financiamientos deben dirigirse a investigación sobre "enfermedades o problemas de salud," y no necesariamente a una disciplina científica.

Sin mermar su posición discursiva positiva hacia la $M G$, subrayaron que es necesario enfrentar otros problemas que forman parte del "rezago epidemiológico" 2 de los sistemas de salud de México.

.....Mientras no alcancemos la cobertura de salud a nivel nacional, mientras no tengamos a la población educada y acceso a los servicios de salud con adecuada alimentación, entrenamiento físico y todas las medidas preventivas, no podemos aplicar la MG (entrevistada E11M).

En cuanto a las aplicaciones, los investigadores de la categoría opinan que los primeros resultados se verán directamente en la farmacogenómica y la nutrigenómica. Un entrevistado conocía el "Mammaprint", una prueba genómica para medir el riesgo de metástasis en mujeres caucásicas con diagnóstico de cáncer de mama y que ayuda a tomar decisiones en la fase de quimioterapia. En 2007, la Food and Drug Administration (FDA) aprobó el uso de esta prueba en los EU.

Hay pocas [aplicaciones] al momento pero hay algunas muy claras, por ejemplo, la prueba genómica que ya hay para predecir qué tanto un tumor de mama tiene posibilidades de ser un tumor de mal pronóstico o de buen pronóstico. Esto surge de estudios genómicos en Holanda en un grupo de pacientes muy grande y se ha validado en estudios mucho mayores... ya se ha generado una prueba comercial que permite tomar decisiones de cirugías y terapias mucho más severas o menos severas en mujeres con cáncer de mama... (entrevistada E5M).

\section{Factibilidad política: financiamiento $y$ actores institucionales}

Varios de los entrevistados en esta categoría opinaron que es prioritaria la inversión y el financiamiento en $M G$, mientras otros consideraron que no es una prioridad, aunque debe financiarse como un área en desarrollo. 
Pues no es un área prioritaria ... de nuestro país; sin embargo, es un área que no se debe dejar descubierta porque podríamos pagar muy caro el costo de no aplicar investigación en MG a largo plazo (entrevistado E8H).

Subrayaron el papel del Conacyt como un organismo que debe tener la visión de financiar proyectos de investigación en genómica y, además, deben participar otros sectores, como el privado, en la inversión para un desarrollo eficiente de esta área emergente. El Inmegen se percibe como la forma en que el Estado y el Congreso de la Unión procuran el financiamiento para la investigación en genómica.

Sí, por supuesto que sí,... es considerado....por el Congreso de la Unión, por las instituciones que otorgan financiamiento como el Conacyt, por los mismos empresarios [quienes] están conscientes de la necesidad de entender el GH de una manera mas profunda para poder hacer este tipo de prevención y tener una visión más a futuro en cuanto a nuestra individualidad como mexicanos (entrevistado E12H).

\section{Promesas infladas}

\section{Aplicabilidad y aplicaciones de la medicina genómica}

Los entrevistados opinaron que es una exageración afirmar que por medio de la MG se llegará a una mejoría en la práctica clínica (discurso de las 3 "p" exagerado) y que las expectativas generadas por la promesa de la MG están "infladas", sobre todo en los objetivos de mejorar la prevención en SP. Los investigadores señalaron que la investigación en genómica es lo importante, ya que hablar de MG confiere una posible aplicabilidad que requiere de muchos pasos en un proceso de validación científica y clínica.

Aun con todo el esfuerzo por la secuenciación del genoma $\mathrm{y}$ todos estos proyectos internacionales que han seguido al genoma humano, todavía estamos en el estadío en que la mayoría de los genes y las variantes de esos genes que condicionan la mayoría de las enfermedades comunes no se conocen, es decir, todavía estamos en el periodo de diseñar o establecer estrategias distintas para encontrar esos genes. Entonces es una etapa de investigación básica que tiene que desarrollarse (entrevistada E3M).

Para hablar de la generación de productos y servicios es importante conocer las características genómicas de los mexicanos en comparación con otras poblaciones, y uno de los entrevistados comentó que llegar a la medicina personalizada es una "promesa interesante:"
Bueno, lo que se dice es que conocer el genoma de los mexicanos, si es que se pudiera llegar a ello, permitiría adelantar cuáles serían las principales causas de enfermedad y de muerte y, con ello, introducir medidas preventivas....bueno, ya sabemos que muchas de ellas ya están presentes en la actualidad como la obesidad, como la diabetes, como el cáncer (...) la medicina genómica nos permitiría tener una idea más concreta, ya no a nivel de población sino a nivel de individuos; entonces creo que es una promesa interesante (entrevistado E1H).

Los entrevistados en esta categoría no tienen conocimiento de pruebas exitosas en MG porque consideran que la mayoría de las aplicaciones están en etapa de investigación. Indicaron que en el mundo -aunque no en México- se han demostrado algunas aplicaciones en el área de farmacogenómica, e incluso consideraron que no hay aplicaciones que tengan que ver con el diagnóstico de una enfermedad multifactorial.

\section{Factibilidad política: financiamiento y actores institucionales}

Los entrevistados consideraron que es importante invertir en ciencias genómicas, pero que éstas no se pueden contemplar como un área superior a otras. Perciben al Inmegen como una institución que, de manera primordial, tiene que interactuar con los demás Institutos Nacionales de Salud.

Yo creo que es un área prioritaria (para financiamiento) como lo ha venido siendo a lo largo de la administración, pero no debe ser el impedimento de otras áreas igualmente importantes de la atención a la salud como el resto de los Institutos (Nacionales de Salud); yo creo que tiene que ser balanceado (entrevistado E1H).

De manera directa, los entrevistados auguraron que, de no invertirse de forma multidisciplinaria, la información generada por los resultados encontrados sería limitada y reduccionista.

...Esta área necesariamente es multidisciplinaria; si no se alimenta de otras áreas mucho más básicas, realmente no puede tener complementariedad y la informatividad que tendría si nada más se hicieran los aspectos aplicados (entrevistada E3M).

De nuevo se aludió al Conacyt como un organismo clave para el financiamiento, y al sector privado, ejemplificado por la Funsalud, como una agencia financiadora importante. En el caso del Inmegen, se mencionó nuevamente que su posición estratégica debe ser congruente 
con la misión de un instituto de investigación que a la larga podrá generar productos y servicios.

Lo más importante es que se vea esta iniciativa (de la medicina genómica en México) con una solidez realmente de un instituto de investigación, no un instituto de productividad que pueda generar productos o servicios aplicables al mediano o largo plazo; eso no puede ser todavía (entrevistada E3M).

\section{Escepticismo}

En esta categoría se encontró a un investigador con una opinión negativa y con dudas acerca de la posibilidad de que se llegue a encontrar una aplicación generada por la MG. Consideró que la MG ha modificado las creencias en el ámbito académico, pero que no se ha probado un impacto real. Indicó, por otra parte, que en caso de encontrarse una aplicación, el proceso que va del descubrimiento a la traducción hacia la medicina clínica, o a la SP, será de largo plazo.

La medicina genómica realmente puede no impactar si realmente no se concibe cuáles son los problemas reales de salud, y de entrada un problema que la medicina genómica promete resolver pero que va a llevar muchos años y quizás nunca lo logre. Yo no siento que sea una garantía ... es un área que se tiene que explorar, se tiene que contemplar, pero no siento que necesariamente haciéndolo garantiza que te va a dar información para arreglar los problemas de salud ni la diabetes, ni el cáncer de mama, ni el cáncer de pulmón, ni la obesidad, los problemas urinarios, ni nada (entrevistado E9H).

En opinión del investigador, ésta no es un área importante en términos de financiamiento. Desde su punto de vista, antes de seguir invirtiendo en genómica se deben cubrir, en primera instancia, los servicios básicos de salud:

No creo que sea un área prioritaria toda vez que no garanticen servicios básicos de salud a toda la población. Es importante que se dé financiamiento, que se explore esa área a nivel de los problemas prioritarios de salud, pero no como una prioridad porque es prioridad; primero garantizar proveer las cuestiones básicas ... el Estado debe estar más preocupado por garantizar atención primaria y una cobertura (de salud) más amplia a la población en general (entrevistado E9H).

\section{Discusión}

Se diseñó y aplicó un cuestionario para explorar, entre diversos investigadores biomédicos mexicanos, si consideraban la MG como una herramienta con aplicación en SP. Sin embargo, de manera indirecta se identificó en todos los entrevistados una asimilación del discurso de las $3 " p^{\prime \prime}$. En el discurso de los entrevistados se observó que existen posiciones diversas con respecto a las promesas de la MG. Tres de las cuatro categorías mostraron una tendencia positiva en el sentido de que los resultados se verán en dos áreas: la farmacogenómica y la nutrigenómica.

De esta manera, los investigadores visualizan una estrategia nueva con respecto a la prevención secundaria y terciaria; sin embargo, para lograr un impacto en la prevención primaria, donde las acciones están orientadas a detectar grupos susceptibles a ciertas enfermedades, la posibilidad de contar con una aplicación de MG científicamente válida es lejana.

De las dos áreas antes mencionadas, la farmacógenomica se vislumbra como una herramienta que, en el corto plazo, se aplicará en la práctica clínica. Las pruebas mencionadas por los entrevistados fueron aquéllas empleadas en terapia con anticoagulantes (warfarina y acenocumarol) y el "Mammaprint" y que, actualmente, ya se consideran entre las estrategias terapéuticas en el plano internacional.

Uno de los entrevistados, perteneciente a la categoría "promesas infladas", concluyó que para contar con una prueba genética de este tipo, propia para la población mexicana, ésta tendrá que pasar antes por un proceso riguroso de validación clínica.

Con referencia al financiamiento, la inversión y a los actores institucionales, la mayoría de los entrevistados de las primeras tres categorías comentaron que es importante contar con financiamiento para los estudios en MG, aunque hubo divergencia con respecto a considerarlo como una prioridad en el sistema de salud mexicano. Los entrevistados de la categoría "modernindad diferida" observan al Inmegen como expresión de "la buena fe" del Estado y del Congreso de la Unión en esta nueva área del conocimiento. El Inmegen es un proyecto de largo plazo que debe contar con apoyo para lograr el desarrollo de la genómica en México.

El Conacyt es percibido como el órgano rector para la movilización de los recursos federales hacia la investigación y, en general, existe una percepción positiva con respecto a la inversión de la iniciativa privada, como es el caso de Funsalud. Esto indica una posición abierta 
por parte de los investigadores a la posibilidad de que los proyectos de investigación en genómica procedan de un financiamiento público-privado, ${ }^{16}$ lo que incidiría positivamente en la factibilidad de los proyectos, dado que la inversión en esta área es de alto costo. El escéptico tiene una posición radical al no contemplar los beneficios que tendrá la MG; desde su punto de vista, la inversión tendría que destinarse a mejorar los servicios médicos preventivos que ya ofrece el Estado a la población mexicana.

En general, los entrevistados no mostraron preocupación por los aspectos ético-jurídicos que, de manera paralela, se suman a los retos de una traducción eficiente de la investigación genómica en aplicaciones clínicas y de SP. Este resultado probablemente se debió a que la muestra de los entrevistados por criterios de inclusión estuvo formada sólo por investigadores del área biomédica, pues no se entrevistó a ningún investigador de las áreas sociales, éticas o jurídicas relacionadas con la MG. Esto último es importante, dado que los retos ético-jurídicos relacionados con la investigación en ciencias genómicas trascienden los problemas clásicos de la bioética en genética clínica e investigación en seres humanos, cuestión que no se exploró aquí y que constituye una limitación importante del estudio. ${ }^{18-21}$

La implementación de reglamentos y normas en el Sector Salud tiene que considerar detalladamente todos los aspectos relacionados con la naturaleza de este bien público y sus repercusiones en el análisis del costo-beneficio de una política nueva. ${ }^{22,23}$

Otra limitante importante de este estudio es que no se abordó la interrelación de los determinantes sociales de la salud y la dimensionalidad que adquiere la $\mathrm{MG}$ ante los mismos en la definición del proceso saludenfermedad. Esta limitación se da en el contexto en el cual fueron revisadas las entrevistas: el congreso en el que participaron los entrevistados se centraba específicamente en los avances científicos en las áreas biomédicas, haciendo poca alusión a los aspectos ético-sociales relacionados con la MG. En este sentido se observó que, en su discurso, el investigador "escéptico" subrayaba la importancia de los determinantes sociales de los que dependen la mayor parte de las políticas públicas de salud en México.

Las ventajas que ofrece la MG serán factibles en el marco de programas de investigación científica permanentes, que permitan generar nuevos conocimientos genómicos con miras a la socialización de sus aplicaciones. Se reconoce que las promesas de la MG contemplan, a su alrededor, varios retos científicos, ético-jurídicos y sociales, por lo cual habrá que redefinir la temporalidad de las aplicaciones a partir de expectativas realistas. ${ }^{24}$ Los resultados presentados en este artículo son explo- ratorios y tratan de identificar cómo se interrelaciona la MG con su aplicación en la SP. Se considera que será necesario diseñar un nuevo estudio para explorar más a fondo ese fenómeno.

\section{Agradecimientos}

Los autores agradecer al doctor Ernesto Schwartz (Manchester University, School of Social Anthropology, Reino Unido), por su asesoría en la metodología y el análisis cualitativo de este estudio.

Declaración de conflicto de interés: los autores declararon no tener conflicto de interés.

\section{Referencias}

I. Collins FS. Schattuck Lecture - Medical and societal consequences of the Human Genome Project. N Engl J Med I999; 34I (I): 28-37. 2. Collins FS, Green ED, Guttmacher E, Guyer MS. A vision for the future of genomics research. A blueprint for the genomic era. Nature 2003;422:835-847.

3. Jimenez-Sanchez G. Developing a platform for genomic medicine in Mexico. Science 2003;300:295-296.

4. Aréchiga $H$, Drucker-Colín R, Jimenez-Sanchez G, Laclette JP, Lopez de Salines A, Martuscelli J, et al. El proyecto del genoma humano: perspectivas de la medicina genómica. Rev Fac Med UNAM 2000;435: 202-205.

5. Barrera H, Martinez-Garza S, Ortiz-Lopez R. Diagnóstico molecular de cáncer. Rev Invest Clin 2003;55:I28-I37.

6. Kershenobich D. Biología molecular en gastroenterología. Rev Invest Clin 2003;55:181-185.

7. Tusie-Luna MT. El camino hacía la comprensión molecular de las enfermedades. Rev Invest Clin 2003;55:|43-I47.

8. Mutchinick OM. La medicina en un contexto genético y molecular. Rev Invest Clin 2003; 55:186-190.

9. Lisker R. De la hipótesis de la evolución de las especies a la Genómica, pasando por la Genética. Rev Invest Clin 2003; 55: I I0-II8.

10. Salamanca-Gomez F, Carnevale A, Kofman-Alfaro S, Lopez M, Torres Maldonado LC, Armendares S, et al. An update of molecular genetics in medicine. Gac Med Mex 1993; 129:273-289.

II. Silva-Zolezzi I, Hidalgo-Miranda A, Estrada-Gil J, Fernandez-Lopez JC, Uribe-Figueroa L, Contreras A. Analysis of genomic diversity in Mexican Mestizo population to develop genomic medicine in Mexico. Proc Natl Acad Sci U S A 2009; 106: 86 I 1-8616.

12. Jiménez-Sánchez G. Oportunidades para la industria farmacéutica en el Instituto Nacional de Medicina Genómica de México. Gac Med Mex 2002; 1383: 291-294.

13. Seguin B, Essajee S, Jimenez-Sanchez G, Singer P, Daar A. Human genomic variation studies and pharmacogenomics are critical for global health. En: Suarez-Kurtz G, ed. Pharmacogenomics in ddmixed populations. Landes Bioscience 2007.

14. Epstein CJ. Medical genetics in the genomic medicine of the 2 Ist century. Am J Hum Genet 2006;793:434-438.

I5. Hanney SR, Gonzalez-Block MA, Buxton MJ, Kogan M. The utilization of health research in policy-making: concepts, examples and methods of assessment. Health Research Policy and Systems, 2003. [consultado 20II agosto 26] Disponible en: http://www.health-policy-systems.com/ content/I/I/2.

16. Reich MR, Public-private partnerships for public health. Nature Medicine 2000;6:617-620. 
17. Ruiz-Ruiz J. Análisis sociológico del discurso: métodos y lógicas. Forum Qualitative Sozialforschung / Forum: Qualitative Social Research I0(2), Art. 26, 2009.

18. Oliva-Sánchez PF, Zaga-Galante J, Arellano-Méndez A, Millet-Hassey F, Rosenthal-Peretzman J, Isasi R. HumGen en español: una herramienta legislativa y política en genómica humana y salud pública. Rev Panam Salud Publica 20II;29: 475-476.

19. Romero-Hidalgo S, Urraca N, Parra D, Villa AR, Lisker R, Carnevale A. Attitudes and anticipated reactions to genetic testing for cancer among patients in Mexico City. Genet Test Mol Biomarkers 2009;13 :477-483.

20. Lisker R. Medicina genómica, mitos y realidades. Rev Invest Clin 2004; 56:554-560.
21. Lisker R, Carnevale A. Changing opinions of Mexican geneticists on ethical issues. Arch Med Res 2006;37:794-803.

22. Knoppers BM, Scriver C. Genomics, health and society: Emerging issues for public policy. En: The Policy Research Initiative. Canada, 2004 23. Comisión Mexicana de Macroeconomía y Salud. Macroeconomía y Salud. Invertir en salud para el desarrollo económico. México: Fondo de Cultura Económica, Colección Biblioteca de la Salud, 2006.

24. Evans JP, Meslin EM, Marteau TM, Caulfield F. Policy Forum: Deflating the genomic bubble. Science 201 I;331:861-862. 